\title{
The role of quorum sensing signalling in EPS production and the assembly of a sludge community into aerobic granules
}

\author{
Chuan Hao Tan ${ }^{1,2,3}$, Kai Shyang Koh ${ }^{1}$, Chao Xie ${ }^{1}$, Martin Tay ${ }^{1,2,4}$, Yan Zhou ${ }^{2}$, \\ Rohan Williams ${ }^{1}$, Wun Jern $\mathrm{Ng}^{2,3}$, Scott A Rice ${ }^{1,4,5}$ and Staffan Kjelleberg ${ }^{1,4,5}$ \\ ${ }^{1}$ Singapore Centre on Environmental Life Sciences Engineering (SCELSE), Nanyang Technological University, \\ Singapore; ${ }^{2}$ Advanced Environmental Biotechnology Centre (AEBC), Nanyang Environment and Water \\ Research Institute (NEWRI), Nanyang Technological University, Singapore; ${ }^{3}$ The School of Civil and \\ Environmental Engineering, Nanyang Technological University, Singapore; ${ }^{4}$ The School of Biological \\ Sciences, Nanyang Technological University, Singapore and ${ }^{5}$ Centre for Marine Bio-Innovation and School \\ of Biotechnology and Biomolecular Sciences, University of New South Wales, Sydney, Australia
}

\begin{abstract}
Quorum sensing (QS) signalling has been extensively studied in single species populations. However, the ecological role of QS in complex, multi-species communities, particularly in the context of community assembly, has neither been experimentally explored nor theoretically addressed. Here, we performed a long-term bioreactor ecology study to address the links between QS, organization and composition of complex microbial communities. The conversion of floccular biomass to highly structured granules was found to be non-random, but strongly and positively correlated with $\mathrm{N}$-acyl-homoserine-lactone (AHL)-mediated QS. Specific AHLs were elevated up to 100 -fold and were strongly associated with the initiation of granulation. Similarly, the levels of particular AHLs decreased markedly during the granular disintegration phase. Metadata analysis indicated that granulation was accompanied by changes in extracellular polymeric substance (EPS) production and AHL add-back studies also resulted in increased EPS synthesis. In contrast to the commonly reported nanomolar to micromolar signal concentrations in pure culture laboratory systems, QS signalling in the granulation ecosystem occurred at picomolar to nanomolar concentrations of AHLs. Given that low concentrations of AHLs quantified in this study were sufficient to activate AHL bioreporters in situ in complex granular communities, AHL mediated QS may be a common feature in many natural and engineered ecosystems, where it coordinates community behaviour.

The ISME Journal (2014) 8, 1186-1197; doi:10.1038/ismej.2013.240; published online 16 January 2014

Subject Category: Microbial population and community ecology

Keywords: biofilm; granulation; model ecosystem; mixed microbial communities; population; quorum sensing
\end{abstract}

\section{Introduction}

Bacteria in natural habitats predominantly exist as matrix-encased species-rich communities, either as surface associated biofilms or surface independent floccular aggregates. This is in contrast to their planktonic single species population counterparts commonly studied in laboratory conditions. The formation and dispersal of structured bacterial biofilms or aggregates occur in response to a range of

Correspondence: S Kjelleberg, Singapore Centre on Environmental Life Sciences Engineering (SCELSE), Nanyang Technological University, Singapore 637551, Singapore.

E-mail: LASKJELLEBERG@ntu.edu.sg

Received 28 July 2013; revised 30 November 2013; accepted 4 December 2013; published online 16 January 2014 environmental cues and signals, such as changes in nutrient concentrations, oxygen, temperature, as well as chemicals and predatory stresses (Bassler et al., 1993; Matz et al., 2004; McDougald et al., 2011; Mitri et al., 2011). In many cases, the signal transduction pathways, the associated changes in gene expression and the involvement of second messenger systems have also begun to be unravelled (Barraud et al., 2009; Petrova and Sauer, 2012). For example, it is now well appreciated that many bacteria rely on a regulatory system based on the production, secretion and response to signalling molecules, termed quorum sensing (QS), as a mechanism to control biofilm development for bacterial populations and this form of control is also postulated to occur within bacterial communities (Williams et al., 2007). The $N$-acylhomoserine-lactone (AHL) mediated QS system is one 
of the best studied bacterial QS systems and has been shown to regulate virulence factor expression, competition and biofilm formation in many different microorganisms and indeed has recently been found also in Archaea (Zhang et al., 2012).

Quorum sensing research has made significant advances in defining the molecular pathways involved in AHL signalling and activation of QS regulated genes as well as the integration of AHL mediated QS with other global regulators to ensure the proper timing of gene expression (Williams et al., 2007). Although such studies have focused on populations of bacteria in the laboratory, bacteria in natural and man-made environments mainly exist as phylogenetically highly diverse and complex communities (Kolter and Greenberg, 2006). These communities are unlike those of population derived assemblages, with community members differentially experiencing fluctuations in a wide range of parameters, including $\mathrm{pH}$, temperature, nutrient concentration, oxygen level and diffusion/dilution of signals (Boyer and Wisniewski-Dyé, 2009; Decho et al., 2009; Yeon et al., 2009). Despite these challenges, it is now increasingly clear that QS occurs in and appears to be highly relevant for complex communities, whether they are biofilms on rocks in streams (McLean et al., 1997), stromatolites (Decho et al., 2009) or biofilms formed on hollow fibre membranes in bioreactors (Yeon et al., 2009). For example, the concentration of AHLs in a microbial mat was shown to vary with the diel cycle, driven by $\mathrm{pH}$ changes as a function of community metabolism and photosynthesis (Decho et al., 2009). However, the role of QS signalling in microbial communities remains poorly understood both in terms of how the signals may impact on community assembly and the function or interaction of the community members.

A microbial granulation system was used in this study as a model ecosystem for complex microbial community assembly. Granulation is an emerging nutrient removal biotechnology, which enables decentralized low energy, cost effective and sustainable water treatment (Liu and Tay, 2004). Our granulation ecosystem has many advantages in that key process parameters, including dissolved oxygen concentrations, $\mathrm{pH}$, temperature, carbon, nitrogen and phosphorus concentrations, as well as the conversion of the floccular biomass into dense, highly structured granules can be precisely controlled and quantified. Acquiring this metadata set allows for the simultaneous and integrated investigation of environmental factors impacting on the production, accumulation, maintenance and functional role of AHL signals in the microbial community. Using this model ecosystem and a combination of analytical chemistry, metatranscriptomics and ecology study approaches, we have investigated the role of AHL based QS signalling in the assembly and morphogenesis of a highly complex granular sludge community.

\section{Materials and methods}

\section{Bioreactor operation}

A sequencing batch reactor, seeded with floccular sludge community (Zhou et al., 2010), was operated for 154 days at $22^{\circ} \mathrm{C}$ under conditions selecting for the formation of microbial granules (Supplementary Materials and Methods). The bioreactor operation involved a $6 \mathrm{~h}$ cycle with two continuous phases of feeding, anaerobic, aerobic and anoxic periods. A total of $2 \mathrm{l}$ synthetic wastewater (Zhou et al., 2010) was fed to the bioreactor during the feeding stages and $2 \mathrm{l}$ of treated effluent was discharged at the end of the cycle, giving a hydraulic retention time of $12 \mathrm{~h}$. The granulation process was monitored on a weekly basis. Concentrations of sludge biomass, including mixed liquor suspended solids and mixed liquor volatile suspended solids, as well as concentrations of ammonia, nitrite, nitrate and orthophosphate were determined using APHA standard engineering methods (Eaton et al., 2005). Sludge particle size was determined using a laser particle size analysis system (Malvern Mastersizer, Singapore). Morphology of the sludge particles was observed under a dissecting microscope (Olympus SZX2-ILLT, Singapore). Sludge volumetric index at 5 min $\left(\mathrm{SVI}_{5}\right)$, which measures sludge density/compactness, was determined as described by Liu (2008). One millilitre aliquots of mixed sludge suspension were collected by the end of the anoxic stage. The sludge biomass was collected at $8000 \mathrm{~g}$ for $5 \mathrm{~min}$ and stored at $-80^{\circ} \mathrm{C}$ immediately for subsequent RNA and extracellular polymeric substances (EPS) analysis. Similarly, $50 \mathrm{ml}$ aliquots of treated effluent were kept at $-80^{\circ} \mathrm{C}$ immediately after sampling for later extraction of AHLs.

\section{Chemicals}

The following synthetic AHLs (>97\%) were purchased from Sigma-Aldrich (Singapore): N-butyryl-DL-homoserine lactone (C4-HSL), N-hexanoyl-DL-homoserine lactone (C6-HSL), $\mathrm{N}$-(3-oxohexanoyl)-DL-homoserine lactone (3OC6-HSL), $N$-heptanoyl-DL-homoserine lactone (C7-HSL), N-octanoyl-DL-homoserine lactone (C8-HSL), $\quad N$-(3-oxooctanoyl)-L-homoserine lactone (3OC8-HSL), $N$-decanoyl-DL-homoserine lactone (C10HSL), N-(3-oxodecanoyl)-L-homoserine lactone (3OC10HSL), $N$-dodecanoyl-DL-homoserine lactone (C12-HSL), $\mathrm{N}$-(3-oxododecanoyl)-L-homoserine lactone (3OC12HSL), $N$-(3-hydroxydodecanoyl)-DL-homoserine lactone (3OHC12-HSL), $N$-tetradecanoyl-DL-homoserine lactone (C14-HSL) and $N$-(3-oxotetradecanoyl)-Lhomoserine lactone (3OC14-HSL).

\section{Detection of AHLs by high performance liquid chromatography/tandem mass spectrometry (HPLC-MS/MS)}

Extraction of AHLs from treated effluents (bulk liquid/ sludge supernatants) were conducted as described by Shaw et al. (1997). Briefly, $50 \mathrm{ml}$ of the supernatants 
were extracted twice with two volumes of dichloromethane. The dichloromethane extracts were concentrated and resuspended in $50 \mu \mathrm{l}$ of methanol: water $(1: 1 \mathrm{v} / \mathrm{v})$ before analysis by HPLC-MS/MS (Shimadzu, Singapore). All samples were chromatographed by HPLC (XR-ODS C18 column) at a flow rate of $0.3 \mathrm{ml} \mathrm{min}^{-1}$. The mobile phase consisted of a linear gradient (40-95\%) of solvent B (methanol with $0.1 \%$ formic acid) and solvent A (25 mM ammonium formate with $0.1 \%$ formic acid). Effluents were ionized by electrospray ionization under positive mode and detected using the multiple reaction monitoring approach (Morin et al., 2003; Decho et al., 2009). Matrix-matched multiple reaction monitoring experiments were conducted for all 13 standard AHLs (Supplementary Table S1). Each standard multiple reaction monitoring profile, including specific LC retention time, appearance of precursor ion $\mathrm{m} / \mathrm{z}$ and two transition ions, as well as relative intensity of the two transition ions, was used as a reference. To confirm the identity of the putative AHLs, a full scan, ranging from $\mathrm{m} / \mathrm{z} 100$ to 350 , coupled with precursor ion scan mode was conducted in comparison with the standards. The extraction efficiency for each AHL was estimated based on the recovery of the standards at two different concentrations, 5 and $50 \mu \mathrm{gl}^{-1}$, added to the heat-inactivated sludge supernatant sample matrix.

\section{Quantification of AHLs by HPLC-MS/MS}

For AHL quantification, matrix-matched standard curves, ranging from 0.5 to $200 \mu \mathrm{g} \mathrm{l}^{-1}$, were constructed. Two transition ions, characteristic of the respective AHLs, were used to identify the AHL and the transition ion with the highest intensity was employed to construct the standard curves (Supplementary Table S1). The analyte peak areas were integrated using LabSolutions (Shimadzu). The limits of detection and quantification for each AHL were calculated with a signal-to-noise ratio of 3.3 and 10, respectively. Triplicate blank injections were performed between sample injections to avoid sample carry over.

\section{EPS analysis}

Extraction of EPS from the sludge biomass was performed as described by Adav and Lee (2007), with some modifications. Briefly, $1 \mathrm{ml}$ of mixed sludge suspension was collected at $8000 \mathrm{~g}$ for $5 \mathrm{~min}$. The sludge biomass was ground gently with a clean glass rod to disrupt the granular particles and subjected to freeze-drying for $24 \mathrm{~h}$. The dried biomass was recorded. The samples were resuspended in $1 \mathrm{ml}$ of $0.9 \% \mathrm{NaCl}(\mathrm{w} / \mathrm{v})$ solution with $0.6 \%$ formaldehyde $(\mathrm{v} / \mathrm{v})$ for $1 \mathrm{~h}$ at $4{ }^{\circ} \mathrm{C}$. Four hundred microliters of $1 \mathrm{M} \mathrm{NaOH}$ were added to the sludge suspension for $3 \mathrm{~h}$ at $4{ }^{\circ} \mathrm{C}$ before collecting EPS at $17000 \mathrm{~g}$ for $15 \mathrm{~min}$ at $4^{\circ} \mathrm{C}$. The polysaccharide (PS) component of EPS was measured using the phenol-sulfuric acid assay with glucose as the standard (Dubois et al., 1956), whereas the protein (PN) component was quantified by the bicinchoninic acid assay with bovine serum albumin as the standard (Smith et al., 1985).

\section{AHL add-back studies}

Two liters of floccular sludge, used to seed the granulation bioreactor, was collected (Zhou et al., 2010). The sludge biomass was washed three times with $0.9 \% \mathrm{NaCl}$ solution before the exposure of fresh synthetic wastewater. Forty mililiters of the sludge suspension were distributed to the individual flasks, followed by the addition of $40 \mu \mathrm{l}$ exogenous AHLs, for example, C6-HSL, C8-HSL, C12-HSL, 3OC6-HSL, 3OC8-HSL and 3OC12-HSL, to a final concentration of 100 or $5000 \mathrm{~nm}$. DMSO was included as the negative control. For each treatment, three biological replicates were performed. The culture flasks were incubated at $100 \mathrm{r}$.p.m. for $1 \mathrm{~h}$ at $22^{\circ} \mathrm{C}$. Two aliquots of $1 \mathrm{ml}$ mixed sludge suspension were sampled from each flask at time 0 and $1 \mathrm{~h}$. The sludge biomass was collected at $8000 \mathrm{~g}$ for $5 \mathrm{~min}$ and snap-frozen with liquid nitrogen before the storage at $-80^{\circ} \mathrm{C}$ for further EPS analysis. Measurements of mixed liquor suspended solids and mixed liquor volatile suspended solids were taken for each flask at the beginning and the end of the experiment. Two-way ANOVA and Bonferroni tests were conducted for statistical analysis.

\section{Metatranscriptomic studies}

Total RNA was extracted from sludge using FastRNA Pro Soil-Direct kit (MP Biomedicals, Singapore) according to the manufacturer's guidelines and DNA was removed using the TURBO DNA-free kit (Applied Biosystems, Singapore). RNA quality was determined using Agilent 2100 Bioanalyser and reported as RNA integrity number. The quantity of total RNA and residual DNA was measured by Quant-iT RiboGreen RNA and PicoGreen DNA assays (Invitrogen, Singapore), respectively. Total RNA, $200 \mathrm{ng}$, was used for complimentary DNA (cDNA) library preparation according to the manufacturer's instructions (Illumina, Singapore). Each cDNA library was ligated with a unique adaptor sequence for sample multiplexing. A total of 16 different cDNA libraries were pooled and sequenced by Miseq System (Illumina).

\section{Sequence analysis}

Total RNA sequencing data were analyzed using a fast tag-based method developed here. This PCR free approach has the advantage of minimizing amplification bias (Logares et al., 2013). Briefly, a universal primer (CGACRRCCATGCANCACCT) for the $16 \mathrm{~S}$ 
rRNA hypervariable region (V6) was used to scan each sequencing read to obtain 33 nucleotide (nt) sequences downstream of the primer, and the $33 \mathrm{nt}$ sequences are defined as the V6 tag of the 16S rRNA gene. Short reads not able to cover the full $33 \mathrm{nt}$ region were discarded. The universal primer matches $94 \%$ of $16 \mathrm{~S}$ rRNA sequences in the RDP database (Claesson et al., 2010). Each different V6 tag is used as a signature sequence to represent one operational taxonomic unit. To eliminate sequencing error derived V6 tags, only V6 tags that were observed in at least two different reads were kept for analysis, and V6 tags were removed when any single type of sequencing read accounted for $\geqslant 50 \%$ of all reads covering that $\mathrm{V} 6$ tag to eliminate sequencing artifacts where identical duplicated reads were generated from single templates.

\section{Statistical analysis}

All statistical analyses were performed using Prism (GraphPad) or R (www.r-project.org). ANOVAs, Bonferroni and Tukey tests were conducted to examine differences between various granulation phases and adjusted $P$-values were reported.
Pearson correlation coefficients were determined and false discovery rate corrections were made for all multiple correlations and adjusted $P$-values were reported. The correlation matrixes were clustered using unsupervised hierarchical clustering method based on Euclidean distance with complete linkage as coded by Cluster 3.0 and visualized using Java TreeView.

\section{Results}

Development of a complex, functional granular sludge community

Granules are defined as compact aggregates that are characterized with a minimum particle size of $100 \mu \mathrm{m}$ in diameter and have a $\mathrm{SVI}_{5}$ (a measure of biomass density/compactness) of $50 \mathrm{mlg}^{-1}$ or less (Barr et al., 2010). Samples were collected weekly for image analysis, quantification of $\mathrm{SVI}_{5}$, particle size and sludge biomass to monitor the granulation process, and five distinct phases of granular development were defined (Figure 1). At the point of inoculation, the floccular biomass had a mean particle diameter of $60 \mu \mathrm{m}$ (50th percentile distribution).

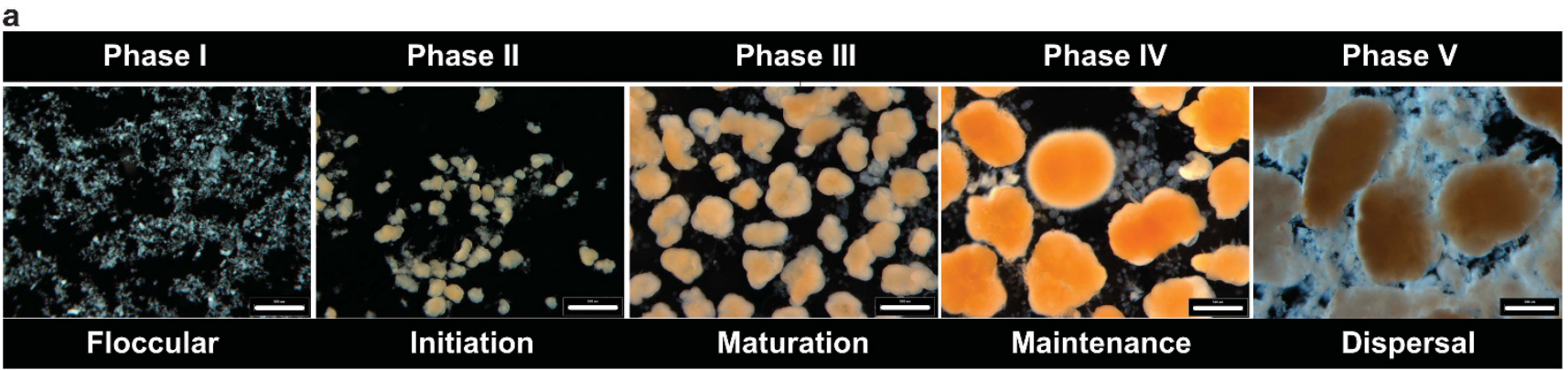

b

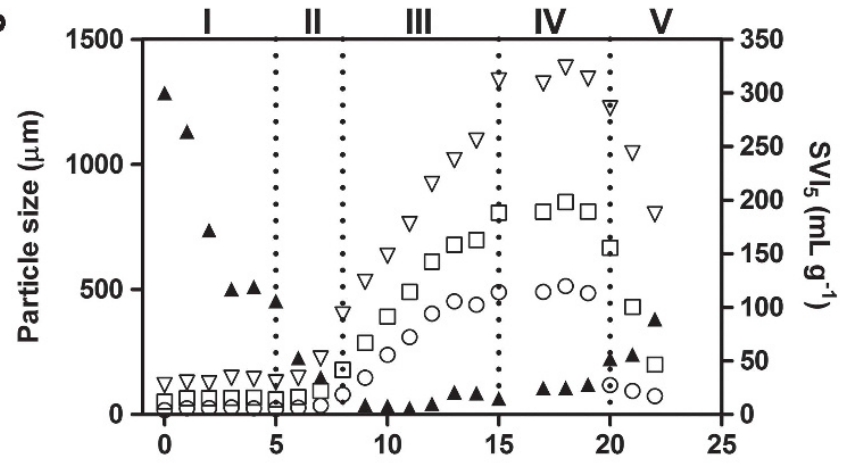

- Sludge volumetric index $\left(\mathrm{SVl}_{5}\right)$

○ 10th percentile

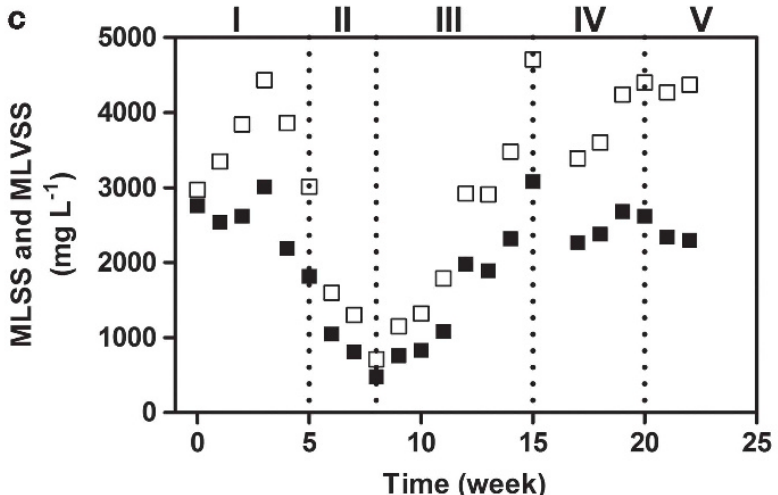

$\square$ Concentration of sludge biomass in terms of MLSS

- Concentration of sludge biomass in terms of MLVSS

Figure 1 Developmental process of granular sludge community in a sequencing batch reactor based on microscopic visualization of structures (a), particle size distribution (measured on a volume basis) and $\mathrm{SVI}_{5}$ (b), and sludge biomass concentration (c). Magnification in (a) $\times 15$ (bar, $500 \mu \mathrm{m}$ ). In (b), 10th percentile (open circle), 50th percentile (open square) and 90th percentile (open triangle) indicate that $10 \%, 50 \%$ and $90 \%$ of total particles were below the corresponding size distribution, respectively; while SVI $_{5}$ (filled triangle) measures the compactness of the sludge particles. Concentration of sludge biomass (c), in terms of mixed liquor suspended solids (open square) and mixed liquor volatile suspended solids (filled square), were also determined. The dotted line separates the different developmental phases of granulation, that is, phases I-V. 
This was followed by the reduction of the sludge settling-time from 60 to $5 \mathrm{~min}$ to select for high density sludge biomass, where a $40 \%$ loss in biomass (mixed liquor volatile suspended solids decreased from 3000 to $1820 \mathrm{mgl}^{-1}$ ) and a $70 \%$ reduction in biomass volume $\left(\mathrm{SVI}_{5}\right.$ decreased from 300 to $106 \mathrm{mlg}^{-1}$ ) were observed (phase I; weeks $0-5$ ). Compact aggregates developed in the bioreactor by week 8 as the mean particle size increased from 60 to $180 \mu \mathrm{m}$ and $\mathrm{SVI}_{5}$ decreased markedly to $<50 \mathrm{mlg}^{-1}$ (phase II; weeks 5-8). This suggests that the biomass was undergoing granulation. Weeks 8-15 (phase III) were characterized by a dramatic increase in the mean particle size from 180 to $850 \mu \mathrm{m}$. Granule size remained steady at $850 \mu \mathrm{m}$ for the subsequent 5 weeks (phase IV; weeks 15-20). At week 20, the mature granules disintegrated into floccular biomass with the mean particle size decreased to $200 \mu \mathrm{m}$ and $\mathrm{SVI}_{5}$ increased to $89 \mathrm{mlg}^{-1}$ (phase V; weeks 20-22), thus completing the granule life cycle. In terms of reactor performance, removal of total nitrogen and phosphorus were rather erratic during the transition from phase I to II where loss of biomass occurred. However, stable and high removal efficiency for both nitrogen (that is, $85 \pm 7 \%$ ) and phosphorus (that is, $95 \pm 11 \%$ ) were achieved once the compact aggregates were formed and increased in biomass at phase IV. Although the phosphorus removal efficiency was maintained during the dispersal phase (phase V), removal of total nitrogen became unstable at this point (that is, $76 \pm 25 \%$ ).

\section{AHL concentration positively correlates with granulation}

The specific AHLs and their concentrations in the mixed microbial community during granulation were determined from sludge supernatant extracts by HPLC-MS/MS. Identification and quantification were based on standard curves and the mass spectra of 13 different synthetic AHLs as quantitative references (Supplementary Figure S1). The extraction efficiency for each of the 13 standards was experimentally determined (between $50 \%$ and $80 \%$ ), and was factored into the quantification of the AHLs. AHLs ranging from C4 to C8 were detected at different stages of the granulation process, whereas AHLs with 10 carbons or more were not resolved within the detection limit $(0.2-2$ pmolg ${ }^{-1}$ after sample concentration and biomass normalization) (Figure 2a). 3OC8-HSL was the most abundant signal, 5- to 30-fold higher than the other AHLs. The amount of 3OC8-HSL in the sludge supernatant, after biomass normalization, was estimated to be $6-325 \mathrm{pmolg}^{-1}$ (Figure 2a), or 10-210 pM equivalent (Supplementary Figure S2). The amount of C4-C8 AHLs, per gram of sludge biomass, increased 10- to 100-fold during phase II as compared with phase I $(P<0.05$ for all AHLs $)$ (Figure 2a), corresponding with the initiation of granule formation (Figure 1). When the relationship between AHLs and granulation at different developmental phases was determined using Pearson correlation, all AHLs were strongly and positively correlated with sludge particle size (as a granulation measure) during the phase I-II transition $(r>0.8567$, $P<0.007$; minimum ' $r$ ' and maximum ' $P$ ' values for all AHLs) (Figure 2b). The concentration of some AHLs, for example, C4-HSL and C6-HSL, continued to increase during phase III, whereas other AHLs, for example, 3OC6-HSL, C7-HSL, C8-HSL and 3OC8HSL, decreased 5- to 10-fold during the same phase (Figure 2a). A second rise in all AHL levels was observed at the maintenance phase (phase IV) and the levels of AHLs declined or were below the detection limit at the time when the granules started to disperse (phase V) (Figure 2a). The amounts of C6-HSL and C8-HSL, in particular, were found to decrease significantly (C6-HSL: $23.0 \pm 0.2$ vs $2.0 \pm 1.3 \mathrm{pmolg}^{-1}, P=0.0015$; C8-HSL: $3.0 \pm 0.2$ vs $0.03 \pm 0.0 \mathrm{pmol} \mathrm{g}^{-1}, P=0.0037$ ) (Figure 2a) and were strongly correlated with the sludge particle size during granule disintegration or phase IV-V transition (C6-HSL: $\quad r=0.7983, \quad P=0.0550 ; \quad$ C8-HSL: $r=0.7670, P=0.0714$ ) (Figure 2b). The levels of the remaining AHLs, for example, C4-HSL, C7-HSL, 3OC6-HSL and 3OC8-HSL, also declined during the dispersal period but the differences were not significant $(P>0.05)$ (Figure 2a). Thus, high concentrations of AHLs were associated with granule formation and low levels of particular AHLs were associated with granule collapse (Figure 2b).

Expression of EPS correlates with granulation and AHL production

EPS, a key component of biofilms and granules, was characterized and quantified based on polysaccharide and protein content. Both polysaccharides and proteins increased in amount as the floccular biomass began to transform into granules (phase I-II; polysaccharides: $13.2 \pm 2.5$ vs $27.3 \pm$ $8.1 \mathrm{mgg}^{-1}, \quad P<0.0001$; proteins: $72.4 \pm 8.5$ vs $110.4 \pm 13.7 \mathrm{mgg}^{-1}, P<0.0001$ ) (Figures 3a and b). The amount of protein showed a slight decline in phase III and remained relatively stable thereafter $\left(100.1 \pm 4.4 \mathrm{mg} \mathrm{g}^{-1}\right.$ ) (Figure 3b). In contrast, the amount of polysaccharides declined during phases IV and $\mathrm{V}$, returning to the initial concentration observed for the floccular sludge $(21.1 \pm 1.1$ vs $13.9 \pm 2.3 \mathrm{mg} \mathrm{g}^{-1}, P=0.0452$ ) (Figure 3a). Consistent with the polysaccharide profile, the polysaccharide to protein ratio (PS/PN) increased during phase I-II transition $(0.18 \pm 0.02$ vs $0.24 \pm 0.04, P=0.0070)$ and decreased during phase IV-V transition $(0.22 \pm 0.01$ vs $0.13 \pm 0.03, P=0.0025$ ) (Figure 3c). For the latter transition, the PS/PN ratio decreased to $0.13 \pm 0.03$, a ratio that was comparable with the EPS composition of the floccular biomass. The PS/PN ratio showed a strong positive Pearson correlation with the sludge particle size, in particular during phase I-II (floccular to granular phase; $r=0.8950$, 

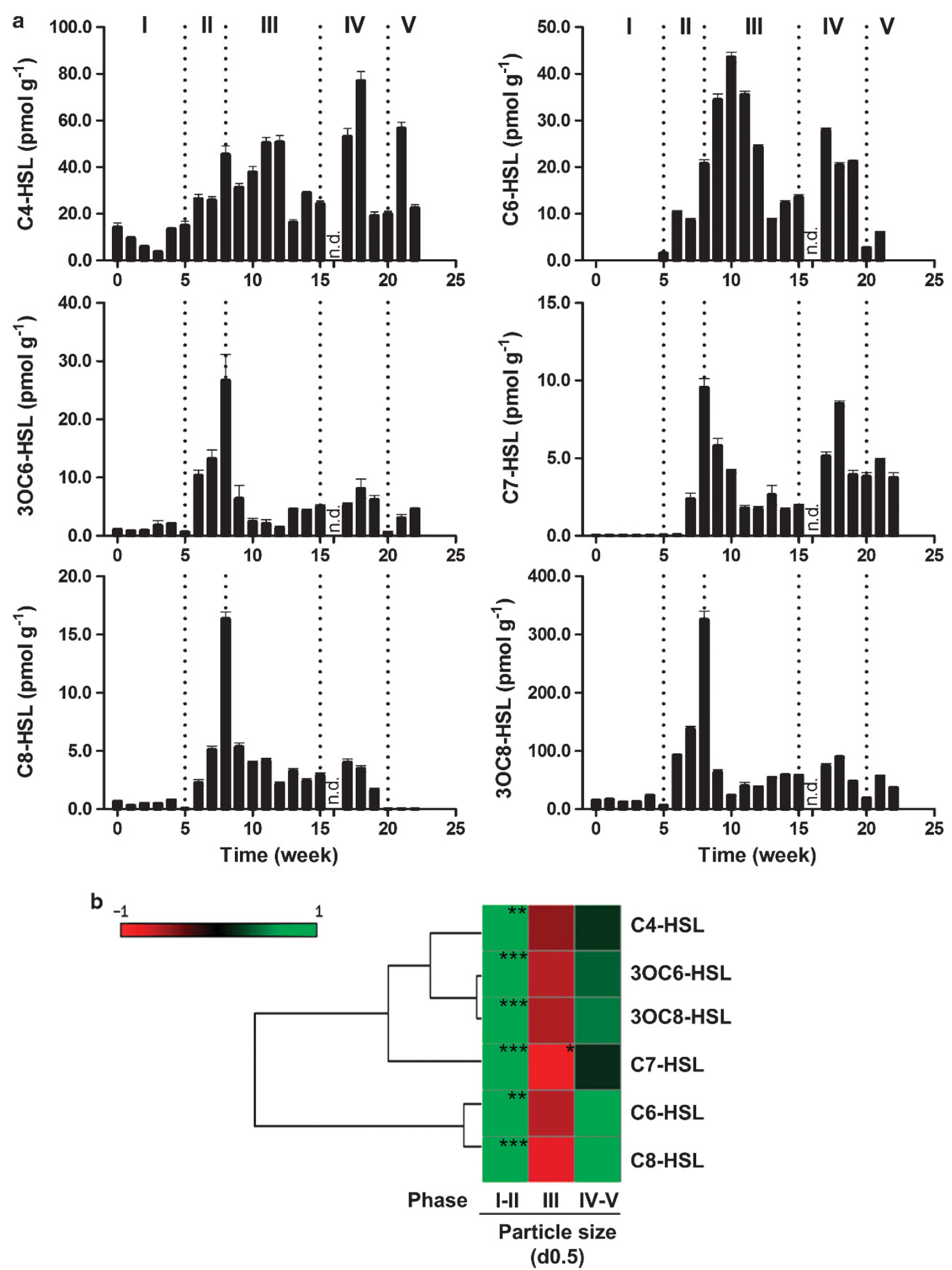

Figure 2 The expression of AHLs correlates with different developmental phases of granulation. (a) HPLC-MS/MS profiling of AHLs extracted from the sludge supernatant during granulation. The identity and quantity of individual AHLs present in each sample were compared with the multiple reaction monitoring profiles of 13 standard AHLs. A total of six AHLs are shown (the remaining seven AHLs were below the detection limit). The data were normalized to the respective sample biomass. Error bars are defined as s.e.m. ( $n=3$, technical replicates). The dotted line separates the different developmental phases of granulation, that is, phases I-V while n.d. represents 'not determined'. (b) Unsupervised clustering of Pearson correlations between individual AHLs and sludge particle size (d0.5 or 50th percentile distribution) at specific phase transitions. False discovery rate (FDR) corrections for multiple comparisons were performed and significant differences are indicated as follows: ${ }^{*} P<0.05,{ }^{* *} P<0.01$ and ${ }^{* * *} P<0.001$. 

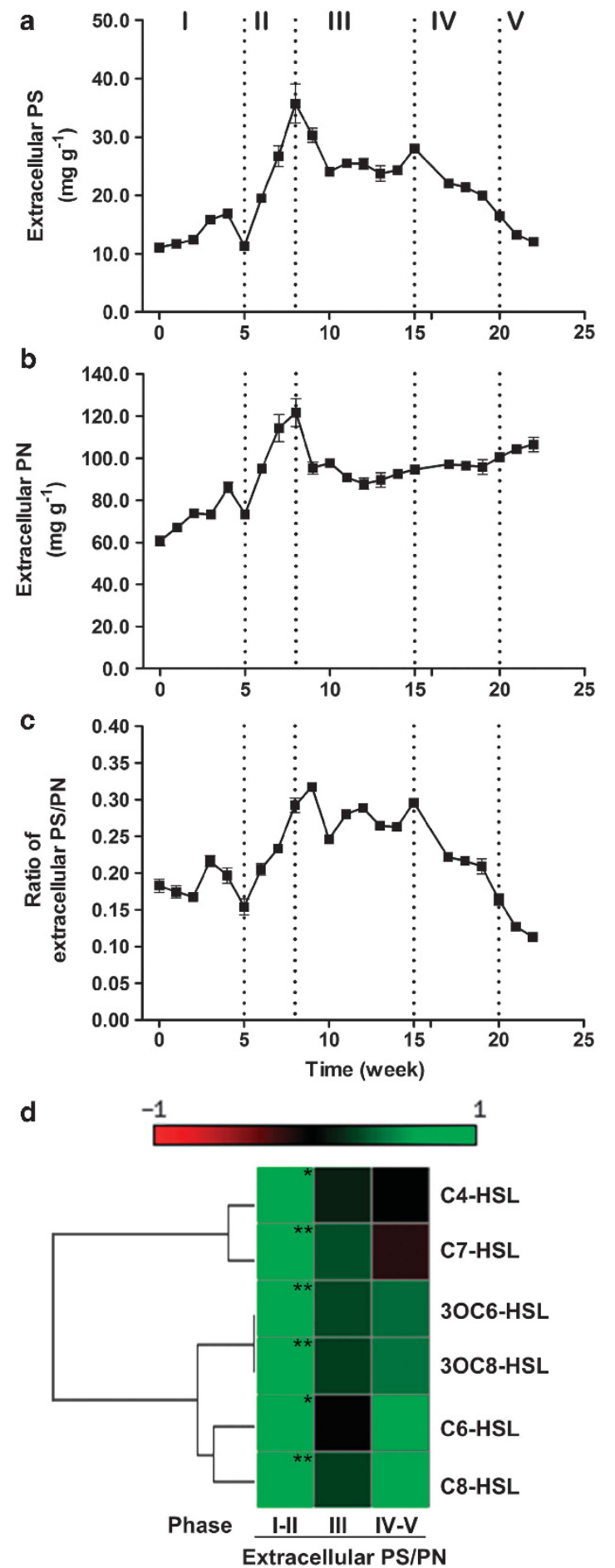

Figure 3 The expression of EPS correlates with granulation and AHL production at different developmental stages. Total EPS was extracted from the sludge biomass and quantified for the extracellular polysaccharides (PS) (a) and extracellular proteins (PN) (b) components. Each EPS component was normalized to the respective sample biomass. The ratio of extracellular polysaccharide to protein (PS/PN) was also determined (c). Error bars are defined as s.e.m. ( $n=3$, technical replicates). The dotted line separates the different developmental phases of granulation, that is, phases I-V. Unsupervised clustering of Pearson correlations between PS/PN ratio and the individual AHLs (d). FDR corrections for multiple comparisons were performed and significant differences are indicated as follows: ${ }^{*} P<0.05$ and ${ }^{*} P<0.01$.
$P=0.0033$ ) and phase IV-V (granular to floccular phase; $r=0.8357, P=0.0288$ ) transitions. Not only did the EPS content correlate with the development and dispersal of microbial granules, but it was observed that the PS/PN ratio also correlated with AHL accumulation in the bioreactor (Figure 3d). The production of all AHLs and the PS/PN ratio were statistically correlated during the phase I-II transition $(r>0.7899, P<0.0339$; minimum ' $r$ ' and maximum ' $P$ ' values for all AHLs), whereas C8-HSL was the only signal that strongly correlated with the PS/PN ratio during phase IV-V transition $(r=0.7921, P=0.0867)$ (Figure $3 \mathrm{~d})$.

AHL addition induces EPS production in the floccular sludge community

The exogenous addition of 3OC6-HSL, 3OC8-HSL and 3OC12-HSL, as well as the unsubstituted C6-HSL, significantly increased the production of both extracellular polysaccharides $(14-36 \%, P<0.05$ for all indicated AHLs) and proteins $(7-16 \%, P<0.05$ for all indicated AHLs) by the floccular sludge community, whereas C8-HSL and C12-HSL had no effect on the total EPS production after $1 \mathrm{~h}$ of incubation (Figures 4a and b). Moreover, the production of extracellular polysaccharides was found to be induced in a signal concentration-dependent manner (100 and $5000 \mathrm{nm;} \mathrm{F}=26.58, P<0.0001$ ) (Figure 4a). For example, addition of 3OC6-HSL to the floccular sludge at 100 and $5000 \mathrm{~nm}$ was found to increase the production of polysaccharides by $18 \%$ and $36 \%$, respectively. Addition of these AHLs (that is, 3OC6HSL, 3OC8-HSL, 3OC12-HSL and C6-HSL) also resulted in an increased PS/PN ratio, from 0.21 to $0.24(P<0.05$ for all indicated AHLs) (Figure 4c), for the floccular sludge, which was consistent with the PS/PN ratio of the granular sludge (Figure 3c).

Community composition correlates with AHL production and granulation

Analysis of total rRNA sequences using the Shannon-Weaver index, a measure of community richness and evenness, revealed a community shift from high to low diversity during the initiation of granulation (phase I-II; $11.42 \pm 3.81$ vs $8.85 \pm 1.48$, $P=0.2612$ ) followed by a subsequent increase in diversity during the maintenance phase (phase III-IV; $7.85 \pm 0.82$ vs $14.40 \pm 1.14, P=0.0070$ ) (Supplementary Table S2 and Supplementary Figure S3). To relate the changes in microbial species abundance and community evenness to granulation and AHL concentration, Pearson correlation coefficients were calculated by comparing the top 50 most abundant community members with the concentration of individual AHLs as well as the stage of granulation. The microbial community members were divided into three clusters based on their correlation with AHL concentration and granulation (Figure 5). Community members in cluster 1 showed no correlation to AHLs or granulation. Members in 

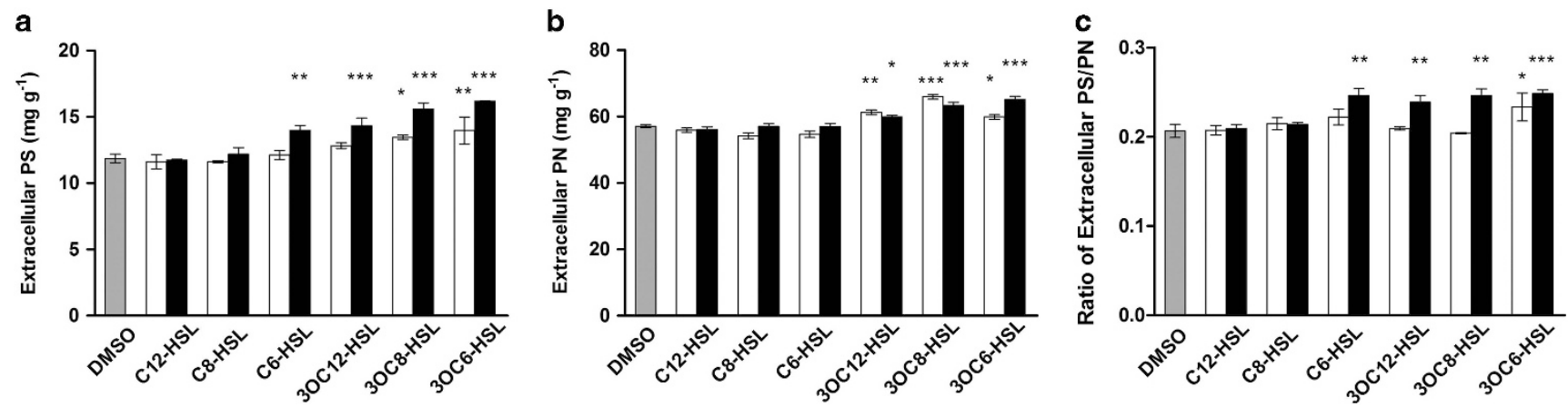

Figure 4 Add-back of specific AHLs to the floccular sludge drives EPS production. Six different synthetic AHLs were separately added to the microscosms at two different concentrations, $100 \mathrm{~nm}$ (open bar) and $5000 \mathrm{~nm}$ (filled bar), for $1 \mathrm{~h}$ with constant shaking at $100 \mathrm{r}$.p.m. DMSO was used as a solvent control (grey bar). Expression of extracellular polysaccharides (PS) (a) and extracellular proteins (PN) (b) were assessed $1 \mathrm{~h}$ post incubation. Each EPS component was normalized to the respective sample biomass. The ratio of extracellular polysaccharide to protein (PS/PN) expressed is depicted (c). Error bars are defined as s.e.m. ( $n=3$, biological replicates). Two-way ANOVA was performed and Bonferroni post-tests were conducted to compare each treatment to the negative control where significant differences are indicated as follows: ${ }^{*} P<0.05,{ }^{*} P<0.01$ and ${ }^{*} * * P<0.001$.

cluster 2, which accounted for $>50 \%$ of the top 50 most abundant community members, demonstrated a strong positive correlation with at least one AHL as well as with granulation. A Xanthomonadaceae bacterium (Tag 6), representing cluster 2, was isolated from the granular sludge and was demonstrated to produce AHLs ranging from C6 to C10, with C7-HSL being the dominant signal (Supplementary Figures S4a and c). Microorganisms in cluster 3 were negatively correlated with granulation as well as AHL concentration. Interestingly, a cluster 3 member, of the Comamonadaceae family (Tag 17), was isolated and found to be an AHL signal degrader (Supplementary Figures S4b and d).

\section{Discussion}

Quorum sensing signalling represents an intriguing area of research especially with respect to the behaviour and ecology of microbial assemblages. Many details surrounding signal coordinated expression of phenotypes have been elucidated for single species microbial populations. However, with few exceptions, bacteria exist in the environment as mixed communities of organisms and it remains unclear how QS functions in such high diversity communities. Here, we have begun to address the community level QS signalling and responses using a microbial granulation bioreactor system, comprised of $\sim 500$ operational taxonomic units (data not shown), as our experimental ecosystem.

Despite the signals being present at concentrations that were 5-200 times lower than those found for many pure culture systems (Pearson et al., 1995; Burton et al., 2005; Schaefer et al., 2008), the AHLs were physiologically relevant and could be detected in situ using a fluorescent bioreporter strain (Supplementary Figure S5). Such low, yet active, concentrations have been reported also for two novel QS systems, aryl-homoserine-lactones (Ahlgren et al.,
2011) and branched acyl-homoserine-lactones (Lindemann et al., 2011). These have been found to respond to signal concentrations as low as $10 \mathrm{pm}$. Moreover, it was also demonstrated that endogenous levels of AHLs were less than $20 \mathrm{pm}$ in soil (Wang and Leadbetter, 2005), suggesting that the concentration of extracellular signals in natural environments may be lower than is typically observed for laboratory batch cultures. Although the role of QS remains largely unknown in most natural and engineered habitats, this study has clearly established a strong positive correlation between AHL based QS and formation of microbial granules. Similarly, the reduction in signal concentrations correlated with disintegration of the granules (Figures 1 and 2). It is thus tempting to speculate that QS signalling may have a key role in the granulation process by mediating the communication between different sludge particles for cellular aggregation or are involved in the regulation of community behaviour associated with granular physiology.

The low signal concentrations in the bulk liquid may represent an equilibrium concentration, where the signals, which primarily partition to the biofilm, diffuse out of the sludge into the bulk liquid. It has been proposed that diffusion rates and local concentrations of signals matter more than bulk AHL concentrations, a concept termed diffusion sensing or efficiency sensing (Redfield, 2002; Hense et al., 2007; Dulla and Lindow, 2008). If the local AHL concentrations around the QS organisms can reach a threshold level before subsequently being diluted away into the bulk liquid, then the community may still be able to efficiently employ QS. Although it was not possible to precisely determine the signal concentration accumulated within the sludge biofilms using HPLC-MS/MS, we have semi-quantitatively measured the amount of signals extracted from the floccular sludge biomass using a thin layer chromatography overlaid with a biosensor strain (data not shown). The dominant signal, 3OC8-HSL, was present in the floccular biomass at $>20 \mathrm{~nm}$, 


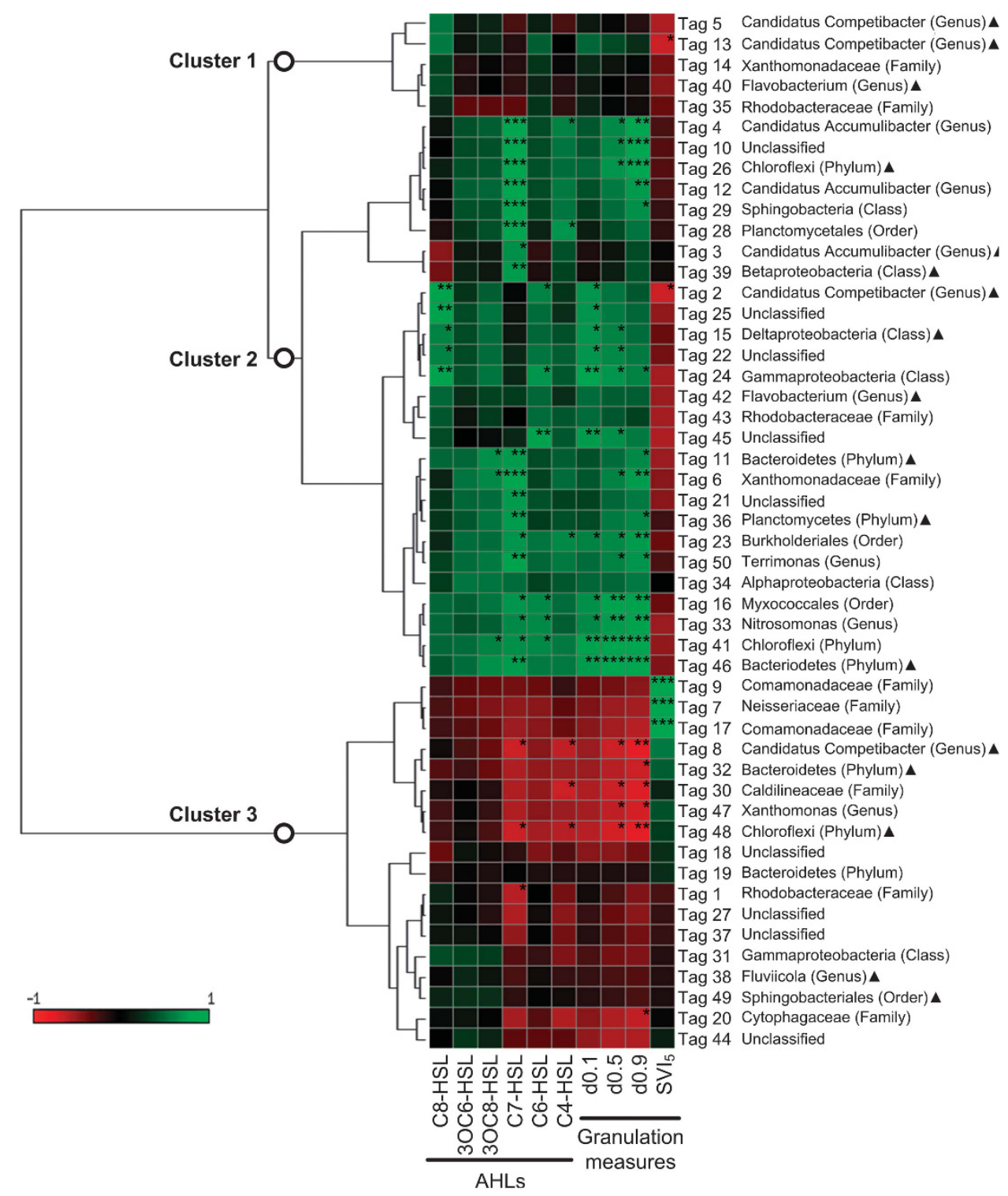

Figure 5 Unsupervised clustering of the top 50 community members in relation to the expression of AHLs and granulation. The relationships between the abundance of the top 50 most dominant community members (each represented by a unique V6 tag), and the concentration profile of AHLs as well as the granulation measures over a time series of 22 weeks (16 time points in total) were calculated based on Pearson correlation. Granulation determinants assessed include particle size distribution (d0.1, 10th percentile; d0.5, 50th percentile; d0.9, 90th percentile) and $\mathrm{SVI}_{5} .{ }^{\mathbf{\Delta}}$ Unclassified based on the taxonomy classification pipeline stated in Materials and methods. Instead, the closest relative with sequence identity matching more than $97 \%$ is shown where it is applicable. FDR corrections for multiple comparisons were performed and significant differences are indicated as follows: ${ }^{*} P<0.05,{ }^{*} P<0.01$ and ${ }^{* * *} P<0.001$.

which was at least 100 -fold higher than the signal concentration in the bulk liquid at the same time point. These observations are consistent with a previous report showing that AHLs preferentially partition to the biofilm, reaching concentrations 600 -fold in excess of the signal concentration in the surrounding bulk liquid medium (Charlton et al., 2000). Thus, it is highly likely that QS induction would be active in the floccular and granular biomass tested here, and may be important in the development of microbial granules.

Previous studies have shown that AHL mediated QS is important for biofilm development, and that in some species this is mediated partly through regulation of EPS production (Davies et al., 1998; Koutsoudis et al., 2006). In addition, the development of granules has been associated with the production of the polysaccharide component granulan (Seviour et al., 2011). It was interesting to note that in this study EPS production also showed a strong positive correlation with AHL concentration and granulation, suggesting an interrelationship of these processes (Figure 3). Although it is not possible to disentangle cause and effect from these data, the increased EPS production on the exogenous addition of AHLs to microcosms of floccular 
sludge may support the hypothesis that the AHLs, which were most abundant in the bioreactor community (3OC6-HSL, 3OC8-HSL and C6-HSL), are involved in the regulation of EPS production. The increased EPS production is unlikely to simply reflect either selection of species that produce EPS, as the experiments were conducted over a short time frame $(1 \mathrm{~h})$, or a consequence of the community utilizing the AHLs as a nutritional source, given that the EPS expression was only induced by specific AHLs (Figure 4). Although the amount of EPS induced by specific AHLs in the batch culture was not as extensive as observed in the natural granulation course, the EPS induction was statistically significant (AHLs vs DMSO control; Polysaccharides: $F=19.68, P<0.0001$; Proteins, $F=43.92, P<0.0001)$ and likely to be of biological importance. The low level of EPS induction observed in these add-back experiments could be a consequence of the short exposure time to AHLs (1 h). Alternatively, optimal EPS production during granulation may require a combination of AHL mediated regulation and additional, unidentified environmental factors or cues.

On the basis of total RNA sequencing, the abundance of more than $50 \%$ of the top 50 community members were positively correlated with AHL concentration across the 22-week experiment (Figure 5: cluster 2). Many of the genera identified here have previously been shown to be involved in AHL mediated QS (Burton et al., 2005; d'AngeloPicard et al., 2005; Suárez-Moreno et al., 2010). Organisms in this group, for example, Nitrosomonas, Burkholderiales and Xanthomonadaceae, have previously been demonstrated to be AHL producers (Burton et al., 2005; d'Angelo-Picard et al., 2005; Suárez-Moreno et al., 2010) and we directly determined that a member of this cluster, for example, Tag 6 (Lysobacter brunescens), as well as a member of cluster 1, for example, Tag 14 (Stenotrophomonas sp.) from the family Xanthomonadaceae, produced AHLs (Supplementary Figures S4a and c). Although not all of the bacteria identified in cluster 2 may be direct contributors to the QS signal pool, they nonetheless likely have important roles in the expression of QS dependent phenotypes. For example, it has been shown that Escherichia coli, which does not produce AHLs, can respond to AHLs via the orphan AHL receptor SdiA, leading to specific changes in gene expression (Hughes et al., 2010). Moreover, a phylogenetic survey by Case et al. (2008) demonstrated that 45 out of 512 complete genomes contained incomplete QS circuits, with homologues of the AHL receptor gene luxR but not the AHL synthase gene luxI. In our system, it was observed that several members of two dominant genera in the community, that is, Candidatus Competibacter and Candidatus Accumulator, previously demonstrated to be essential for granulation through the production of granulan associated with the EPS (Seviour et al., 2011), were positively correlated with QS and granulation (cluster 2), suggesting that they may participate in, or benefit from, QS even though they have not yet been demonstrated to produce AHL signals or carry AHL receptor homologues.

Although it is not yet possible to determine if QS signalling is a direct cause of granulation, the observation here that the three processes, EPS production, community composition changes and AHL concentrations, were all strongly correlated with each other suggests that they are important in the granulation process. This combination of observations suggests that QS may have a role in restructuring of the community, potentially through the regulation of EPS. Here, AHL production may lead to induction of EPS synthesis and hence granulation. Alternatively, the changes in QS signals may be a consequence of community selection during the granulation process. Presumably, this could be mediated by physical removal of low density sludge biomass during the floccular phase (phase I), and the enrichment/selection of specific bacterial populations was reflected in the reduction of community diversity in phases II and III (Supplementary Figure S3). When stable aggregates were formed, the diversity increased along with the sludge biomass (Figure 1; phase III-IV). The increased diversity during phase III could in part be explained by the expansion of populations that can exploit the different micro niches, for example, anoxic/anaerobic and aerobic zones, that form within granules (Meyer et al., 2005). Interestingly, although the same operational parameters were used throughout the experiment, the granules dispersed at phase V (Figure 1). Whereas, dispersal may be related to cell lysis induced by predators, for example, phages (Barr et al., 2010), the dispersal phenomenon was clearly linked to a decrease in AHL concentration in this study (Figure 2). The loss of signals could be the consequence of decreased competiveness of cluster 2 organisms, which were closely associated with QS signalling, resulting in a reduction in AHL controlled EPS production.

Despite the high complexity of the granulation ecosystem, it is clear that AHL mediated signalling and community assembly are linked in the system described here and hence QS is relevant for complex microbial communities. Signal concentrations were at least 100 times higher in the biomass, and were in the ranges previously shown to induce bioreporter strains, suggesting that QS is most relevant within the structured floccular or granular community. We submit that the dynamic physiological conditions experienced by the microbial community in the granular biofilm reactor would not be dissimilar from environmental conditions experienced by many other complex communities in a wide range of natural and bioprocess systems, such as microbial mats, anaerobic digesters, membrane bioreactors and river streams. It is therefore likely that QS signalling has an important functional roles and 
mediates cooperative microbial community behaviour, in particular within biofilm structures.

\section{Conflict of Interest}

The authors declare no conflict of interest.

\section{Acknowledgements}

We thank Peter Little for reading the manuscript and Peter Steinberg for help with the statistical analysis. Special acknowledgement to Zhaoqi Zhan and Peiting Zeng, for help with AHL quantification, and Shimazdu Singapore for providing the use of HPLC-MS/MS. CH Tan was supported by National Research Foundation Singapore under its National Research Foundation (NRF) Environmental and Water Technologies (EWT) PhD Scholarship Programme, administered by the Environment and Water Industry Programme Office (EWI). This work was funded by NRF and Ministry of Education Singapore under its Research Centre of Excellence Programme.

\section{References}

Adav SS, Lee DJ. (2007). Extraction of extracellular polymeric substances from aerobic granule with compact interior structure. J Hazard Mater 154: 1120-1126.

Ahlgren NA, Harwood CS, Schaefer AL, Giraud E, Greenberg EP. (2011). Aryl-homoserine lactone quorum sensing in stem-nodulating photosynthetic Bradyrhizobia. Proc Natl Acad Sci USA 108: 7183-7188.

Barr JJ, Cook AE, Bond PL. (2010). Granule formation mechanisms within an aerobic wastewater system for phosphorus removal. Appl Environ Microbiol 76: $7588-7597$.

Barraud N, Schleheck D, Klebensberger J, Webb JS, Hassett DJ, Rice SA et al. (2009). Nitric oxide signaling in Pseudomonas aeruginosa biofilms mediates phosphodiesterase activity, decreased cyclic di-GMP levels, and enhanced dispersal. J Bacteriol 191: 7333-7342.

Bassler BL, Wright M, Showalter RE, Silverman MR. (1993). Intercellular signalling in Vibrio harveyi: sequence and function of genes regulating expression of luminescence. Mol Microbiol 9: 773-786.

Boyer M, Wisniewski-Dyé F. (2009). Cell-cell signalling in bacteria: not simply a matter of quorum. FEMS Microbiol Ecol 70: 1-19.

Burton EO, Read HW, Pellitteri MC, Hickey WJ. (2005). Identification of acyl-homoserine lactone signal molecules produced by Nitrosomonas europaea strain Schmidt. Appl Environ Microbiol 71: 4906-4909.

Case RJ, Labbate M, Kjelleberg S. (2008). AHL-driven quorum-sensing circuits: their frequency and function among the Proteobacteria. ISME J 2: 345-349.

Charlton TS, de Nys R, Netting A, Kumar N, Hentzer M, Givskov $\mathrm{M}$ et al. (2000). A novel and sensitive method for the quantification of $N$-3-oxoacyl homoserine lactones using gas chromatography-mass spectrometry: application to a model bacterial biofilm. Environ Microbiol 2: 530-541.
Claesson MJ, Wang Q, O’Sullivan O, Greene-Diniz R, Cole JR, Ross RP et al. (2010). Comparison of two next-generation sequencing technologies for resolving highly complex microbiota composition using tandem variable 16S rRNA gene regions. Nucleic Acids Res 38: e200.

d'Angelo-Picard C, Faure D, Penot I, Dessaux Y. (2005). Diversity of $N$-acyl homoserine lactone-producing and -degrading bacteria in soil and tobacco rhizosphere. Environ Microbiol 7: 1796-1808.

Davies DG, Parsek MR, Pearson JP, Iglewski BH, Costerton JW, Greenberg EP. (1998). The involvement of cell-to-cell signals in the development of a bacterial biofilm. Science 280: 295-298.

Decho AW, Visscher PT, Ferry J, Kawaguchi T, He L, Przekop KM et al. (2009). Autoinducers extracted from microbial mats reveal a surprising diversity of $\mathrm{N}$-acylhomoserine lactones (AHLs) and abundance changes that may relate to diel pH. Environ Microbiol 11: 409-420.

Dubois M, Gilles KA, Hamilton JK, Rebers PA, Smith F. (1956). Calorimetric method for determination of sugar and related substances. Anal Chem 28: 350-356.

Dulla G, Lindow SE. (2008). Quorum size of Pseudomonas syringae is small and dictated by water availability on the leaf surface. Proc Natl Acad Sci USA 105: 3082-3087.

Eaton AD, Franson MAH, Association APH, Association AWW, Federation WE. (2005). Standard Methods for the Examination of Water and Wastewater. American Public Health Association: Washington, DC, USA.

Hense BA, Kuttler C, Müller J, Rothballer M, Hartmann A, Kreft JU. (2007). Does efficiency sensing unify diffusion and quorum sensing? Nat Rev Microbiol 5: 230-239.

Hughes DT, Terekhova DA, Liou L, Hovde CJ, Sahl JW, Patankar AV et al. (2010). Chemical sensing in mammalian host-bacterial commensal associations. Proc Natl Acad Sci USA 107: 9831-9836.

Kolter R, Greenberg EP. (2006). Microbial sciences: the superficial life of microbes. Nature 441: 300-302.

Koutsoudis MD, Tsaltas D, Minogue TD, von Bodman SB. (2006). Quorum-sensing regulation governs bacterial adhesion, biofilm development, and host colonization in Pantoea stewartii subspecies stewartii. Proc Natl Acad Sci USA 103: 5983-5988.

Lindemann A, Pessi G, Schaefer AL, Mattmann ME, Christensen QH, Kessler A et al. (2011). Isovalerylhomoserine lactone, an unusual branched-chain quorum-sensing signal from the soybean symbiont Bradyrhizobium japonicum. Proc Natl Acad Sci USA 108: 16765-16770.

Liu Y, Tay JH. (2004). State of the art of biogranulation technology for wastewater treatment. Biotechnol $\mathrm{AdV}$ 22: 533-563.

Liu Y. (2008). Wastewater Purification: Aerobic Granulation in Sequencing Batch Reactors. Taylor \& Francis: Boca Raton.

Logares R, Sunagawa S, Salazar G, Cornejo-Castillo FM, Ferrera I, Sarmento H et al. (2013). Metagenomic 16S rDNA Illumina tags are a powerful alternative to amplicon sequencing to explore diversity and structure of microbial communities. Environ Microbiol; e-pub ahead of print 18 September 2013; doi:10.1111/ 1462-2920.12250.

Matz C, Bergfeld T, Rice SA, Kjelleberg S. (2004). Microcolonies, quorum sensing and cytotoxicity 
determine the survival of Pseudomonas aeruginosa biofilms exposed to protozoan grazing. Environ Microbiol 6: 218-226.

McDougald D, Rice SA, Barraud N, Steinberg PD, Kjelleberg S. (2011). Should we stay or should we go: mechanisms and ecological consequences for biofilm dispersal. Nat Rev Microbiol 10: 39-50.

McLean RJ, Whiteley M, Stickler DJ, Fuqua WC. (1997). Evidence of autoinducer activity in naturally occurring biofilms. FEMS Microbiol Lett 154: 259-263.

Meyer RL, Zeng RJ, Giugliano V, Blackall LL. (2005). Challenges for simultaneous nitrification, denitrification, and phosphorus removal in microbial aggregates: mass transfer limitation and nitrous oxide production. FEMS Microbiol Ecol 52: 329-338.

Mitri S, Xavier JB, Foster KR. (2011). Social evolution in multispecies biofilms. Proc Natl Acad Sci USA 108: 10839-10846.

Morin D, Grasland B, Vallée-Réhel K, Dufau C, Haras D. (2003). On-line high-performance liquid chromatography-mass spectrometric detection and quantification of $N$-acylhomoserine lactones, quorum sensing signal molecules, in the presence of biological matrices. J Chromatogr A 1002: 79-92.

Pearson JP, Passador L, Iglewski BH, Greenberg EP. (1995). A second $N$-acylhomoserine lactone signal produced by Pseudomonas aeruginosa. Proc Natl Acad Sci USA 92: 1490-1494.

Petrova OE, Sauer K. (2012). Dispersion by Pseudomonas aeruginosa requires an unusual posttranslational modification of BdlA. Proc Natl Acad Sci USA 109: 16690-16695.

Redfield RJ. (2002). Is quorum sensing a side effect of diffusion sensing? Trends Microbiol 10: 365-370.

Schaefer AL, Greenberg EP, Oliver CM, Oda Y, Huang JJ, Bittan-Banin G et al. (2008). A new class of homoserine lactone quorum-sensing signals. Nature 454: $595-599$.
Seviour TW, Lambert LK, Pijuan M, Yuan Z. (2011). Selectively inducing the synthesis of a key structural exopolysaccharide in aerobic granules by enriching for Candidatus "Competibacter phosphatis". Appl Microbiol Biotechnol 92: 1297-1305.

Shaw PD, Ping G, Daly SL, Cha C, Cronan JEJ, Rinehart KL et al. (1997). Detecting and characterizing $N$-acylhomoserine lactone signal molecules by thin-layer chromatography. Proc Natl Acad Sci USA 94: 6036-6041.

Smith PK, Krohn RI, Hermanson GT, Mallia AK, Gartner FH, Provenzano MD et al. (1985). Measurement of protein using bicinchoninic acid. Anal Biochem 150: 279.

Suárez-Moreno ZR, Devescovi G, Myers M, Hallack L, Mendonça-Previato L, Caballero-Mellado J et al. (2010). Commonalities and differences in regulation of $N$-acyl homoserine lactone quorum sensing in the beneficial plant-associated Burkholderia species cluster. Appl Environ Microbiol 76: 4302-4317.

Wang YJ, Leadbetter JR. (2005). Rapid acyl-homoserine lactone quorum signal biodegradation in diverse soils. Appl Environ Microbiol 71: 1291-1299.

Williams P, Winzer K, Chan WC, Cámara M. (2007). Look who's talking: communication and quorum sensing in the bacterial world. Philos Trans R Soc Lond B Biol Sci 362: 1119-1134.

Yeon KM, Cheong WS, Oh HS, Lee WN, Hwang BK, Lee CH et al. (2009). Quorum sensing: a new biofouling control paradigm in a membrane bioreactor for advanced wastewater treatment. Environ Sci Technol 43: 380-385.

Zhang G, Zhang F, Ding G, Li J, Guo X, Zhu J et al. (2012). Acyl homoserine lactone-based quorum sensing in a methanogenic archaeon. ISME J 6: 1336-1344.

Zhou Y, Ganda L, Lim M, Yuan Z, Kjelleberg S, Ng WJ. (2010). Free nitrous acid (FNA) inhibition on denitrifying poly-phosphate accumulating organisms (DPAOs). Appl Microbiol Biotechnol 88: 359-369.

Supplementary Information accompanies this paper on The ISME Journal website (http://www.nature.com/ismej) 